\title{
FINITE GROUPS WHICH ADMIT A FIXED-POINT-FREE AUTOMORPHISM GROUP ISOMORPHIC TO $S_{3}$
}

\author{
DAVID PARROTT
}

(Received 6 December 1988; revised 23 June 1989)

Communicated by $\mathrm{H}$. Lausch

\begin{abstract}
Let $G$ be a finite group of even order coprime to 3. If $G$ admits a fixed-point-free automorphism group isomorphic to the symmetric group on three letters, then we prove that $G$ is soluble.
\end{abstract}

1980 Mathematics subject classification (Amer. Math. Soc.) (1985 Revision): 20 D 05.

A number of authors (for example, [6], [7], [8]) have shown that finite groups admitting certain fixed-point-free abelian automorphism groups are soluble. In this paper we show that a finite group $G$ which admits a fixed-point-free automorphism group isomorphic to $S_{3}$ (the symmetric group on 3 letters) is soluble if $|G|$ is even and coprime to 3 . A similar result for groups of odd order coprime to 3 has been proved by B. Dolman [1].

The result proved here is a consequence of Glauberman's characterization of simple groups of order coprime to 3 [3]. However the proof given in this paper uses fairly elementary methods and (of course) relies on the fixedpoint-free automorphism group.

Throughout the paper we put

$$
\Sigma=\left\langle\sigma, \pi \mid \sigma^{3}=\pi^{2}=1, \pi \sigma \pi=\sigma^{-1}\right\rangle \cong S_{3} .
$$

Our notation will in general follow Gorenstein's book [4]. In particular, if $P$ is a $p$-group, $J(P)=\langle A| A \subseteq P, A$ is abelian of maximal order $\rangle$. In addition, $J_{e}(P)=\langle E| E \subset P, E$ is elementary abelian of maximal order $\rangle$. The theorem proved in this paper is as follows:

(C) 1990 Australian Mathematical Society 0263-6115/90 \$A2.00+0.00 
THEOREM. Let $G$ be a finite group of even order coprime to 3. Suppose $G$ admits a fixed-point-free group of automorphisms $\Sigma \cong S_{3}$. Then $G$ is soluble and either

(i) $G$ has a normal 2-complement, or

(ii) $G=(T \times O(G)) \cdot C_{G}(\sigma)$, where $T=O_{2}(G)$ is a Sylow 2-subgroup of $G$.

\section{Preliminary results}

Proposition 1 (Burnside, [5, (10.15)]). If the finite group $X$ admits a fixed-point free automorphism of order 3 then $X$ is nilpotent of class at most 2.

Proposition 2 (Dolman [1]). Let $G$ be a finite group, $(|G|, 3)=1$. Suppose $G$ admits a fixed-point-free group of automorphisms $\Sigma \cong S_{3}$. Then $G$ contains a unique $\Sigma$-invariant Sylow $p$-subgroup for all primes $p$ that divide $|G|$. Further, any $\Sigma$-invariant p-subgroup is contained in this unique ¿-invariant Sylow p-subgroup.

Proof. Let $\mathscr{S}=\{P \mid P$ is a $\sigma$-invariant Sylow $p$-subgroup of $G\}$. By [4, Theorem 6.2.2], $\mathscr{S} \neq \varnothing$ and if $P, Q \in \mathscr{S}$ then $P$ and $Q$ are conjugate by some element in $C_{G}(\sigma)$. Clearly $C_{G}(\sigma)$ has odd order as $\pi$ is fixed-pointfree on $C_{G}(\sigma)$. Thus $|\mathscr{S}|=\left|C_{G}(\sigma): N_{G}(P) \cap C_{G}(\sigma)\right|$ is odd $(P \in \mathscr{S})$. As $\pi$ permutes the subgroups of $\mathscr{S}, \pi$ fixes a subgroup of $\mathscr{S}$.

Suppose $P, Q$ are both $\Sigma$-invariant Sylow $p$-subgroups of $G$ such that $P=Q^{x}$ for some $x \in C_{G}(\sigma)$. Thus

$$
Q^{x}=P=\pi(P)=\pi\left(Q^{x}\right)=\pi(Q)^{x^{-1}}=Q^{x^{-1}}
$$

whence $x^{2} \in N_{G}(Q)$. Thus $x \in N_{G}(Q)$ as $x \in C_{G}(\sigma)$ which has odd order. The last part now follows from the fact that the normalizer of a $\Sigma$-invariant $p$-group is also $\Sigma$-invariant.

If the finite group $G$ has order coprime to $3, \operatorname{SL}(2, p)$ cannot be involved in $G$. Hence two consequences of Glauberman's $Z J$-theorem apply for primes $p \geq 5$.

Proposition 3 [2, Corollaries 2.1, 2.2]. Let $p$ be an odd prime which divides $G, G$ a finite group of order coprime to 3 . Let $S$ be a Sylow psubgroup of $G$ and $N=N_{G}(Z(J(S)))$. Then

(i) $G / O^{p}(G) \cong N / O^{p}(N)$; 
(ii) two subsets of $S$ are conjugate in $G$ if and only if they are conjugate in $N$.

The structure of soluble groups of odd order admitting a fixed-point-free group of automorphisms isomorphic to $S_{3}$ has been determined by E. Shult.

Proposition 4 [10, Corollary 2.1]. Let $H$ be a soluble group of order coprime to 6 which admits a fixed-point-free group of automorphisms isomorphic to $S_{3}$. Then $H^{\prime}$ is nilpotent.

The following result (also due to Shult), which plays a key role in the proof of Proposition 4, is used (in this paper) to study soluble groups of even order admitting $\Sigma$ fixed-point-free.

Proposition 5 [9, Theorem 3.1]. Let $p$ be an odd prime and $H$ the semidirect product of the normal subgroup $K$, of order coprime to $p$, and $\langle\rho\rangle$ of order $p$. Suppose $H$ acts faithfully on the elementary abelian q-group $V$, where $(q,|H|)=1$. If $C_{V}(\rho)=1$ then $[\langle\rho\rangle, K]=1$ unless $K$ has $a$ non-abelian Sylow 2-subgroup and $p$ is a Fermat prime.

Proposition 6. Let $\Sigma$ act fixed-point-free on the finite group $G$ of order coprime to 3. Then $C_{G}(\sigma)$ is abelian of odd order and for any subgroup $X \subseteq C_{G}(\sigma)$ we have $N_{G}(X)=C_{G}(X)$. In particular, if $C_{P}(\sigma)=P$ for any Sylow p-subgroup $P$ of $G$, then $G$ has a normal p-complement.

Proof. Since $\pi$ acts fixed-point-free on $C_{G}(\sigma), C_{G}(\sigma)$ is inverted by $\pi$ and is therefore abelian of odd order. Let $N=\left[N_{G}(X), \sigma\right]$, so $[N, \sigma]=N$ as $(|N|, 3)=1$.

By the Three Subgroups Lemma [4, Lemma 2.2.3], $[N, \sigma, X] \subseteq[X, N, \sigma]$ - $[\sigma, X, N]=1$, whence $N \subseteq C_{G}(X)$. As $X \subseteq C_{G}(\sigma), C_{G}(\sigma) \subseteq C_{G}(X)$ and therefore $N_{G}(X)=\left(C(\sigma) \cap N_{G}(X)\right) \cdot N \subseteq C_{G}(X)$ as required. The final statement follows from Burnside's Transfer Theorem [4, Theorem 7.4.3].

Proposition 7. Suppose $\Sigma$ acts fixed-point free on the group $H=V \cdot U$ where $V$ is elementary abelian of order $p^{n}, p \geq 5, U$ is a $\Sigma$-invariant four group and $V=[V, U]$. If $\langle u\rangle=C_{U}(\pi)$ then $\pi$ inverts $C_{V}(u), V=$ $C_{V}(u) \times C_{V}\left(u^{\sigma}\right) \times C_{V}\left(u^{\sigma^{2}}\right)$ and $C_{V}(\sigma)=\left\{v v^{\sigma} v^{\sigma^{2}} \mid v \in C_{V}(u)\right\}$. In particular, $\left|C_{V}(\sigma)\right|=\left|C_{V}(u)\right|=p^{n / 3}$.

Proof. As $[V, U]=V, C_{V}(U)=1$ so the decomposition of $V$ follows from [4, Theorem 5.3.16]. The three factors have the same order as $\sigma$ per- 
mutes the 3 involutions in $U$. As $[\pi, u]=1, \pi$ normalizes $C_{V}(u)$. Since $\pi$ inverts $C_{V}(\sigma), \pi$ must invert $C_{V}(u)$ also.

\section{Groups of even order}

We begin by determining the structure of a soluble group of even order coprime to 3 which admits $\Sigma$ as a fixed-point-free automorphism group.

Proposition 8. Let $M$ be a soluble group of even order coprime to 3 which has a fuxed-point-free group of automorphisms $\Sigma \cong S_{3}$. Then either

(i) $M$ has a normal 2-complement, or

(ii) if $T$ is a Sylow 2-subgroup of $M, T \triangleleft M$ and $M=(T \times O(M)) \cdot C_{M}(\sigma)$.

Proof. Let $S$ be a $\Sigma$-invariant Sylow 2-subgroup of $O_{2^{\prime}, 2}(M)$ so that $M=N_{M}(S) \cdot O(M)$. Since $\sigma$ is fixed-point-free on $S, C_{M}(\sigma)$ covers $N_{M}(S) / C_{M}(S) \cdot S$ by Proposition 5. Proposition 6 and the fact that $C_{M}(S) \subseteq$ $O_{2^{\prime}, 2}(M)$ [4, Theorem 5.3.3] yield that $S=T$.

Suppose now that (i) does not hold, so that $M \neq T \cdot O(M)$. We must show that $T=O_{2}(M)$. As $\Sigma$ is fixed-point-free on $M / O_{2}(M)$ we may assume $O_{2}(M)=1$.

Let $x \in C_{M}(\sigma) \cap N_{M}(T)-O(M)$ with $x^{p} \in O(M)$ for some odd prime $p$. Suppose first that $[x, Z(T)]=1$. Note that $[x, T] \triangleleft T$ and $[x, T] \neq 1$ because $x \notin O(M)$ and $C_{M}(T) \subseteq T \cdot O(M)$. Hence there exists a $\Sigma$ invariant four group $E \subseteq[x, T] \cap Z(T)$. As $O_{2}(M)=1$, there exists a prime $q$ with $Q=O_{q}(M)$ and $[E, Q] \neq 1$. Let $V=[Q / \Phi(Q), E] \neq 1$ (where $\Phi(Q)$ is the Frattini subgroup of $Q)$. As $[x, E]=1$ and $C_{V}(x) \supseteq C_{V}(\sigma)$, it follows from Proposition 7 that $[x, V]=1$. Now $E \triangleleft T$ so $V$ is $T$ invariant and $V=[V, E]=[V, T]$. The three subgroups lemma yields $[T, x, V]=1$, which contradicts $E \subseteq[T, x]$.

We may now suppose that $[x, Z(T)] \neq 1$. Let $F \subseteq \Omega_{1}(Z(T))$ be a minimal $\Sigma\langle x\rangle$-invariant subgroup with $[F, x]=F$. As $O_{2}(M)=1$ there exists a prime $q$ with $[Q, F] \neq 1$ where $Q=O_{q}(M)$. Let $V$ be a minimal $\Sigma\langle x\rangle F$-invariant subgroup of $[F, Q / \Phi(Q)] \neq 1$.

If $W$ is a minimal $F$-invariant subgroup of $V$ then $W$ has $|\langle\sigma\rangle \|\langle x\rangle|=$ $3|\langle x\rangle|$ conjugates under the action of $\langle\sigma\rangle \times\langle x\rangle$ by [4, Theorem 3.4.3]. This implies however that there exists $w \in C_{V}(\sigma)-C_{V}(x)$, against the fact that $C_{G}(\sigma) \subseteq C_{G}(x)$. This completes the proof of the proposition.

Proposition 9. Suppose the dihedral group $D=\langle\pi, x| x^{p}=\pi^{2}=1, \pi x \pi$ $=x^{-1}, p$ an odd prime $\rangle$ acts on the 2-group $T$ of order $2^{n}$ with $C_{T}(x)=1$. 
For any chief factor $V=S / R$ of $T D$ contained in $T$ we have $C_{V}(\pi)=$ $C_{S}(\pi) R / R$. Further, $C_{T}(\pi)=2^{n / 2}$.

Proof. By a result of Suzuki [4, page 328], any involution in $\pi R$ inverts an element of odd order in $D R$. As $C_{T}(x)=1$, it follows from Sylow's theorem that all involutions in $\pi R$ are conjugate in $D R$ and hence in $\langle\pi, R\rangle$.

Now let $s \in S-R$ with $[\pi, s] \in R$. Then $s^{-1} \pi s=\pi r$ for some $r \in R$. We know that $\pi_{\widetilde{R}} \pi r$ so there exists $t \in R$ with st $\in C_{S}(\pi)$. Thus $C_{T}(\pi)$ covers $C_{V}(\pi)$ as asserted.

The final conclusion follows by induction on the length of a chief series for $D T$ and the fact that $\left|C_{V}(\pi)\right|=2^{k / 2}$ if $|V|=2^{k}$ (note that $V$ is elementary and $\left.C_{V}(x)=1\right)$.

We conclude this section with a result on finite groups with Sylow 2subgroups of class at most 2 .

Proposition 10. Suppose the finite group $G$ has Sylow 2-subgroup $T$ of class at most 2. If $N_{G}(Z(T))=N_{G}(T)=T \cdot C_{G}(T)$ then either

(i) $G$ has a normal 2-complement, or

(ii) $T$ contains a normal subgroup $S$ with $T / S$ cyclic and $N_{G}(S) / C_{G}(S) S$ has a non-trivial normal 2-complement. Further if $(|G|, 3)=1$ then $J_{e}(T) \subseteq$ $S$.

Proof. Let $Z=Z(T)$. We have that $N_{G}(Z)=N_{G}(T)=T \times O\left(C_{G}(T)\right)$. If $Z$ is weakly closed in $T$ then Grun's theorem [4, Theorem 7.5.2] states that $N_{G}(Z)^{\prime} \cap T=G^{\prime} \cap T$. Thus $T^{\prime}=T \cap O^{2}(G) \cdot G^{\prime}$ and as $T^{\prime} \subseteq Z$, the Frattini argument yields $N_{G}\left(T^{\prime}\right)=C_{G}\left(T^{\prime}\right)$. It follows that $O^{2}(G) \cdot G^{\prime}$ has a normal 2-complement by Burnside's transfer theorem, and (i) holds.

We now assume that $Z$ is not weakly closed in $T$ and choose $S$ of maximal order such that

$$
Z \neq Z \cdot Z^{g} \subseteq S=T \cap T^{g} \text { for } g \in G-N_{G}(T) .
$$

As $\left\langle Z, Z^{g}\right\rangle \subseteq S$ and $T^{\prime} \subseteq Z$ we have $S \triangleleft\left\langle T, T^{g}\right\rangle$. Put $N=N_{G}(S)$ and $C=C_{G}(S) \cdot S$ and note that $C_{G}(S)=Z(S) \times O\left(C_{G}(T)\right)$. If $h \in N-N_{G}(T)$ then $Z \neq Z \cdot Z^{h} \subseteq S \subseteq T \cap T^{h}$. The maximality of $|S|$ forces $T \cap T^{h}=S$. We use the bar convention for $N / C$ and we have that $\bar{T}$ is an abelian T.I. Sylow 2-subgroup of $\bar{N}$. Now $\bar{N}$ has one class of involutions [4, Theorem 9.1.4] and by Burnside's Lemma [4, Theorem 7.1.1] all involutions of $\bar{T}$ are conjugate in $N_{\bar{N}}(\bar{T})$. As $N_{\bar{N}}(\bar{T})=\bar{T}$ it follows that $\bar{T}$ is cyclic and $\bar{N}$ has a non-trivial normal 2-complement. Finally, if $\langle\bar{t}\rangle=\Omega_{1}(\bar{T}), \bar{t}$ inverts an element $\bar{r}$ of or odd order at least 5 (if 3 does not divide $|\bar{N}|$ ). As $Z \subseteq Z(S)$, 
$C(Z(S))=S \times O\left(C_{G}(T)\right)=C$. Thus $\left|\Omega_{1}(Z(S)): C(t) \cap \Omega_{1}(Z(S))\right| \geq 4$ and $J_{e}(T) \subseteq S$.

\section{Proof of the theorem}

For the rest of the paper, $G$ will denote a finite group of even order coprime to 3, and $\Sigma$ a group of fixed-point-free automorphisms of $G$. Further we let $G$ be a minimal counterexample to the theorem. If $G$ is soluble, the theorem follows from Proposition 8. Thus $G$ is a non-soluble group and therefore all proper $\Sigma$-invariant subgroups of $G$ are soluble.

LEMMA 1. The group $G$ is simple.

Proof. If $N \triangleleft G$ and $N$ is $\Sigma$-invariant, $\Sigma$ is fixed-point-free on $G / N$. Thus as $G$ is a minimal counterexample, so $N=1$ and $G=G_{1} \times G_{2} \times$ $\cdots \times G_{k}$, the $G_{i}$ non-abelian simple groups which are transitively permuted by $\Sigma$. If $\sigma$ normalizes $G_{i}$ for some $i$, then $C(\sigma) \cap G_{i} \neq 1$ by Proposition 1. As $\pi$ inverts $C_{G}(\sigma), \pi$ normalizes $G_{i}$ so $G=G_{i}$ as required. If $\sigma$ permutes $G_{1}, G_{2}, G_{3}$ then $C(\sigma) \cap G_{1} \times G_{2} \times G_{3} \cong G_{1}$ is non-abelian. This contradicts Proposition 6, and the lemma is proved.

Notation. $T$ will denote the (unique) $\Sigma$-invariant Sylow 2-subgroup of $G$ and $M=N_{G}(T)$. Also $Z=\Omega_{1}(Z(T))$.

By Proposition 8, $M$ is a maximal $\Sigma$-invariant subgroup of $G$ and $N_{G}(Z)$ $=M$ also.

The theorem will be proved by determining the structure of $M$ and using this to deduce that $C_{G}(\pi)$ has a normal 2-complement.

LemMA 2. (i) We have $M=(T \times O(M)) \cdot C_{M}(\sigma)$ and $T \times O(M) \neq M$.

(ii) If $H$ is a maximal $\Sigma$-invariant subgroup of $G, H \neq M$, then $H$ has a normal 2-complement.

(iii) If $U$ is any $\Sigma$-invariant four group in $T$ then $C_{G}(U) \subseteq M$.

Proof. (i) By Proposition 8, $M=(T \times O(M)) \cdot C_{M}(\sigma)$. As $T$ has class at most 2 (Proposition 1), $G$ is simple (Lemma 1) and $M=N_{G}\left(J_{e}(T)\right.$ ) ( $M$ is maximal $\Sigma$-invariant), Proposition 10 yields that $M \neq T \cdot C_{G}(T)=$ $T \times O(M)$. (Note that $C_{G}(T)=Z(T) \times O\left(C_{G}(T)\right.$ ) by Burnside's transfer theorem. As $T \triangleleft M, O(M)=O\left(C_{G}(T)\right)$ and so $T \cdot C_{G}(T)=T \times O(M)$.)

(ii) This follows from Proposition 8.

(iii) Suppose that $C_{G}(U) \subseteq H \neq M$ where $H$ is a maximal $\Sigma$-invariant subgroup of $G$. Let $R=H \cap T \supseteq C_{T}(U)$ so that $H=R \cdot O(H)$ (by 
(ii)). If $U \nsubseteq Z$, take $A$ to be maximal abelian in $R$ with $U Z(T) \subseteq A$. Then $A \triangleleft T$ and in any case there exists $A \in S C N(T)$ with $A \leq R$. By a result of Thompson [4, Theorem 8.5.2], $O(H) \cap M \subseteq O(M)$ and so $H \cap M=$ $R \times(O(M) \cap H)$. Since $N_{G}(Z)=M$ and $C_{O(H)}(U) \nsubseteq M$ we have $U \neq Z$ and also $\left[Z, C_{O(H)}(U)\right] \neq 1$. Hence there exists $1 \neq y \in C_{G}(\sigma) \cap\left[Z, C_{O(H)}(U)\right]$ (by Proposition 1).

As $C_{M}(\sigma) \nsubseteq T \times O(M)$ and $C_{G}(\sigma) \subseteq C_{G}(y)$ we have that $C_{G}(y) \nsubseteq H$. By definition, $y \notin M$ so $C_{G}(y) \nsubseteq M$ either.

Let $L$ be a maximal $\Sigma$-invariant subgroup of $G$ containing $C_{G}(y)$. As $\left[U, C_{M}(\sigma)\right] \neq 1$ and $U \subseteq L$ we have $1 \neq\left[L \cap T, C_{M}(\sigma)\right] \subseteq L \cap T$. This contradicts (ii); namely that $L$ has a normal 2-complement. The lemma is proved.

Notation. Let $\mathscr{P}=\{p \mid p$ prime, $p$ divides $|M: T \times O(M)|\}$. For $p \in$ $\mathscr{P}, P_{1}$ denotes the $\Sigma$-invariant Sylow $p$-subgroup of $M ; P_{0}=P_{1} \cap O(M) ; P$ denotes the $\Sigma$-invariant Sylow $p$-subgroup of $G$. Note that $\mathscr{P} \neq \varnothing$ (Lemma 2(i)) and $P_{1} \subseteq P$ (Proposition 2).

Lemma 3. Let $p \in \mathscr{P}$. Then

(i) $P_{1}=P_{0} \cdot C_{P_{1}}(\sigma)$ is abelian;

(ii) $P_{1}$ is not a Sylow p-subgroup of $G$; that is, $P_{1} \neq P$;

(iii) if $P_{0} \neq 1$ then $Z(P)$ is cyclic and $Z(P)^{\#} \subseteq C_{P_{1}}(\sigma)-P_{0}$.

Proof. (i) If $P_{1}^{\prime} \neq 1$ then $N_{G}\left(P_{1}^{\prime}\right) \subseteq M$ (as $T \subseteq N_{G}\left(P_{1}^{\prime}\right)$ and $\left[P_{1}, T\right] \neq$ 1). Thus $P_{1}=P$, a Sylow $p$-subgroup of $G$. Now $N_{M}(Z(J(P)))^{\prime} \cap P \subseteq$ $P_{0} \neq P$, so by Proposition 3(i) and Lemma 1, $N_{G}(Z(J(P)))=N \nsubseteq M$. Since $[T \cap N, P]=1$ (Lemma 2(ii)) and $O(N)^{\prime} \subseteq F(N)$ (Proposition 4), we must have $P=O_{p}(N)$ by Proposition 3(i). However $1 \neq P \triangleleft\langle M, N\rangle=G$ against Lemma 1. Thus $P_{1}$ is abelian and $P_{1}=P_{0} C_{P_{1}}(\sigma)$ by Proposition 8(ii).

(ii) If $P_{0}=1$, the assertion follows from Proposition 6. Suppose $P_{1}=P$, and $P_{0} \neq 1$. Using the same argument as in (i), we see that Proposition 3(i) forces $P=O_{p}(H)$, where $H$ is the maximal $\Sigma$-invariant subgroup of $G$ containing $N_{G}(Z(J(P)))$. As $C_{G}\left(P_{0}\right) \subseteq M$ we have that $F(O(H)) \subseteq$ $M$ and therefore $O(H) \cap M \triangleleft O(H)$ (Proposition 4). Since $[T \cap H, P]=$ 1 , a transfer theorem [4, Theorem 7.4.4] implies that $[O(H), P]=P$; in particular $O(H) \nsubseteq M$. Now $[O(H) \cap M, P] \triangleleft\langle O(H), T\rangle=G$ so that $N_{M}(P)=C_{M}(P)$.

Let $P_{\pi}=C_{P}(\pi)$ and note that $P_{\pi} \subset P_{0} \subseteq O(M)$. By the Frattini argument $N_{G}\left(P_{\pi}\right)=C_{G}\left(P_{\pi}\right) \cdot\left(N_{G}(T) \cap N_{G}\left(P_{\pi}\right)\right)=C_{G}\left(P_{\pi}\right) \cdot N_{M}\left(P_{\pi}\right)$. As $P \subseteq C_{M}\left(P_{\pi}\right)$ we have, in the same way, that $N_{G}\left(P_{n}\right)=C_{G}\left(P_{\pi}\right) \cdot C_{M}\left(P_{\pi}\right) \cdot N_{M}(P)=C_{G}\left(P_{\pi}\right)$. $N_{M}(P)=C_{G}\left(P_{\pi}\right) \cdot C_{M}(P)=C_{G}\left(P_{\pi}\right)$. 
From above, $[O(H), P]=P$, whereas $\left[C_{O(H)}(\sigma), P\right] \subseteq P_{0}$, because $C_{G}(\sigma)$ is abelian and $P=P_{0} C_{M}(\sigma)$. We apply the bar convention to $O(H) / C_{O(H)}(P)$. Therefore we have a $\Sigma$-invariant subgroup $\bar{X} \cong Z_{q} \times Z_{q}$ for some prime $q \neq p$ with $[\bar{X}, \sigma]=\bar{X}$. There exists $x \in C_{O(H)}(\pi)$ with $\bar{X} \in \bar{X}$ and clearly $x \in N_{H}\left(P_{\pi}\right)$. We complete the proof by showing that $\left[x, P_{\pi}\right] \neq 1$.

Let $\tilde{P}$ be a minimal $\Sigma \bar{X}$-invariant subgroup of $[P, \bar{X}] \neq 1$. Suppose that $C_{\tilde{P}}(\bar{x}) \neq 1$. Then $\tilde{P}=C_{\tilde{P}}(\bar{x}) \times C_{\tilde{P}}\left(\bar{x}^{\sigma}\right) \times C_{\tilde{P}}\left(\bar{x}^{\sigma^{2}}\right)$. Since $\pi$ inverts an element $\bar{x}_{1}$ in $\bar{X}$ and $\bar{x}_{1}$ is fixed-point-free on $C_{\tilde{p}}(\bar{x})$, there exists $y \in C_{\tilde{p}}(\bar{x}) \cap C(\pi)$ (clearly $\pi$ normalizes $C_{\tilde{P}}(\bar{x})$ ). However $1 \neq y y^{\sigma} y^{\sigma^{2}} \in C_{\tilde{p}}(\sigma)$ and as $\pi$ inverts $C_{G}(\sigma), y^{\pi}=y^{-1}$, a contradiction. Thus $C_{\tilde{P}}(\bar{x})=C_{\tilde{P}}(x)=1$. Since $[\tilde{P}, \bar{X}]=\tilde{P},[\tilde{P}, \sigma] \neq 1$ so $C_{\tilde{P}}(\pi) \neq 1$. We have that $\left[x, P_{\pi} \cap \tilde{P}\right] \neq 1$ as required.

(iii) Let $P^{*} \neq 1$ be any $\Sigma$-invariant subgroup of $P_{0}$. As $\left[T, P_{1}\right] \subseteq N_{G}\left(P^{*}\right)$ and $1 \neq\left[T, P_{1}\right] \subseteq T$, Lemma 2 (ii) forces $N_{G}\left(P^{*}\right) \subseteq M$. From $P_{1} \neq$ $P$ it follows that $Z(P)^{\#} \subseteq P_{1}-P_{0}$ and as $Z(P)$ is $\Sigma$-invariant, $Z(P) \subseteq$ $C_{P}(\sigma)$. Suppose that $\Omega_{1}(Z(P)) \supseteq\left\langle a_{0}, b_{0}\right\rangle$. Without loss we may assume that $\left[C_{T}\left(a_{0}\right), b_{0}\right] \neq 1$. Now $C_{G}\left(a_{0}\right) \supseteq P$ so $C_{G}\left(a_{0}\right) \subseteq H \neq M, H$ a maximal $\Sigma$-invariant subgroup of $G$. However $1 \neq\left[C_{T}\left(a_{0}\right), b_{0}\right] \subseteq C_{T}\left(a_{0}\right)$ means that $H$ does not have a normal 2-complement. This contradiction (of Lemma 2(ii)) completes the proof of (iii).

Notation. For $p \in \mathscr{P}$ let $\Omega_{1}(Z(P))=\left\langle a_{0}\right\rangle$ if $P_{0} \neq 1$ and if $P_{0}=1$ take $a_{0}$ to be an element of order $p$ in $P_{1}$.

Lemma 4. For $p \in \mathscr{P}$ we have $P_{1}=\langle a\rangle \times P_{0}$, for some element $a \in C_{P_{1}}(\sigma)$ with $\Omega_{1}(\langle a\rangle)=\left\langle a_{0}\right\rangle$.

Proof. Since $Z(P) \cap P_{0}=1$ and $P_{1}=P_{0} C_{P_{1}}(\sigma)$, it is enough to show that $P_{1} / P_{0}$ is cyclic. Suppose to the contrary; so we may choose $b \in C_{P_{1}}(\sigma)-P_{0}$, $b^{p} \in P_{0}$ and $C_{T}(b) \neq 1$. The argument given in the proof of Lemma 3(iii) may be repeated to prove that $C_{T}\left(a_{0}\right)=1$ and $C_{G}(\sigma) \subseteq C_{G}(b) \subseteq M$. In particular, $\left[\sigma, P_{1}\right]=\left[\sigma, P_{0}\right] \neq 1$ by Proposition 6. Thus $\Omega_{1}\left(P_{1}\right) \supseteq$ $\left\langle a_{0}, b_{0}, Y\right\rangle$ with $\left\langle b_{0}\right\rangle=\Omega_{1}(\langle b\rangle)$ and $Y$ a $\Sigma$-invariant subgroup of type $(p, p)$ with $[Y, \sigma]=Y$.

The following remark will be used in the proof:

(*) If $P^{*}$ is any $\Sigma$-invariant subgroup of $P_{0}$ then $N_{G}\left(P^{*}\right) \subseteq M$; and if $d \in C_{P_{1}}(\sigma)-Z(P)$ then $C_{P}(d)=P_{1}$.

(Recall $1 \neq\left[T, P_{1}\right] \subseteq T$. As $\left\langle T, P_{1}\right\rangle \subseteq C_{G}\left(P^{*}\right)$, Lemma 2(ii) yields $N_{G}\left(P^{*}\right) \subseteq M$. If $\left\langle d_{0}\right\rangle=\Omega_{1}(\langle d\rangle)$ then for some $x \in\left\langle d_{0}, a_{0}\right\rangle, C_{T}(x) \neq 1$. As $1 \neq\left[C_{T}(x), a_{0}\right] \subseteq C_{T}(x)$, the same argument yields that $C_{G}(x) \subseteq M$. 
Thus $C_{P}\left(d_{0}\right)=C_{P}(x)=P_{1}=P \cap M$ as required.)

Let $R=N_{P}\left(P_{1}\right) \neq P_{1}$ and note that $\Omega_{1}(Z(R))=\left\langle a_{0}\right\rangle$. Therefore if $y \in$ $\Omega_{1}\left(Z_{2}(R)\right), y$ has at most $p$ conjugates in $R$. Since $\Omega_{1}\left(P_{1}\right) \supseteq\left\langle a_{0}, b_{0}, Y\right\rangle$ it follows from $(*)$ that $\Omega_{1}\left(Z_{2}(R)\right) \subseteq P_{1}$. Now $\sigma$ is fixed-point-free on $R / P_{1}$ so $\left|R: P_{1}\right| \geq p^{2}$. We conclude that $C(\sigma) \cap \Omega_{1}\left(Z_{2}(R)\right)=\left\langle a_{0}\right\rangle$ and $\left|R: P_{1}\right|=p^{2}$. For $x \in R-P_{1},\left|C(x) \cap\left\langle Y, a_{0}, b_{0}\right\rangle\right| \leq p^{2}$ so $P_{1}$ is the unique abelian subgroup of $R$ of its order. Hence $P_{1}$ char $R$ so $R=P$ and $P_{1}$ char $P$.

Let $H$ be a maximal $\Sigma$-invariant subgroup (of $G$ ) containing $N_{G}(Z(J(P))$ ). Since $\left\langle a_{0}\right\rangle=\Omega_{1}(Z(P)), C_{H}\left(a_{0}\right)$ covers $H / O(H)$ by the Frattini argument. Thus $|H|$ is odd as $C_{T}\left(a_{0}\right)=1$. It now follows from Propositions 3(i) and 4 that $P=O_{p}(H)$. Clearly $O_{p^{\prime}}(H) \subseteq M$ as $C_{G}\left(P_{0}\right) \subseteq M$ (by $\left.(*)\right)$.

Suppose that $H=P(H \cap M)=P N_{M}\left(P_{1}\right)$; that is, $N_{M}\left(P_{1}\right)$ covers $H / F(H)$ $\neq 1$. Let $q$ divide $\left|H: C_{H}\left(P_{1}\right)\right|$ and let $\tilde{Q}$ be the $\Sigma$-invariant Sylow $q$ subgroup of $H$. We have $\tilde{Q} \subseteq N_{M}\left(P_{1}\right)$ whence $\tilde{Q} \subseteq Q_{1}$, the $\Sigma$-invariant Sylow $q$-subgroup of $M$. If $Q_{1} \subseteq O(M), 1 \neq\left[N_{M}\left(Q_{1}\right), T\right] \subseteq T$, so $N_{G}\left(Q_{1}\right) \subseteq M$ by Lemma 2(ii). It follows that $Q_{1} \triangleleft M$ (by Propositions $3(\mathrm{i})$ and 4$)$. However this forces $\left[\tilde{Q}, P_{1}\right]=1$ which contradicts the choice of $q$. We have shown that $q \in \mathscr{P}$ and so $Q_{1}$ is abelian. If $Q$ is the $\Sigma$ invariant Sylow $q$-subgroup of $G$, there exists $c \in C_{Q_{1}}(\sigma)$ with $C_{Q}(c) \neq Q_{1}$ by Lemma 3 (if $Q_{0}=O(M) \cap Q_{1} \neq 1$ ) or Proposition 7 (if $Q_{0}=1$ ).

As $\left\langle a_{0}\right\rangle=\Omega_{1}(Z(P)),\left\langle a_{0}\right\rangle \triangleleft H$, so $\left\langle a_{0}\right\rangle \subseteq Z(H)$ since $H / C_{H}\left(a_{0}\right)$ must be cyclic and $a_{0} \in C_{G}(\sigma)$ which is abelian. Now $c \in C_{G}(\sigma) \subset C_{G}\left(a_{0}\right)=H$ so $c \in \tilde{Q}$. Let $\tilde{P}=C_{P}(c)$ and let $L$ be a maximal $\Sigma$-invariant subgroup of $G$ containing $C_{G}(c)$ (note that $M \neq L \neq H$ ). Since $H=N_{G}(Z(J(P))$ ), Proposition 3(ii) yields that $\left\langle a_{0}\right\rangle$ is weakly closed in $P$ and hence in $\tilde{P}$. It follows from $C_{T}\left(a_{0}\right)=1$ and the Frattini argument that $|L|$ is odd. Let $P_{2}=P \cap L$, a Sylow $p$-subgroup of $L$. If $P_{2} \nsubseteq P_{1}, P_{2}$ is non-abelian (as $\left.b \in P_{2}\right)$. However $a_{0} \in P_{2}^{\prime} \subseteq F(L)$ whence $\left\langle a_{0}\right\rangle \subseteq Z(L)$, a contradiction. Hence $P_{2} \subseteq P_{1}$ and $P_{2}$ is abelian. Now $\left\langle a_{0}\right\rangle$ weakly closed in $P_{2}$ forces $N_{G}\left(P_{2}\right) \subseteq H=P(H \cap M)$. Thus $P_{3}=N_{L}\left(P_{2}\right)^{\prime} \cap P_{2} \subseteq P_{0}$, as $N_{L}\left(P_{2}\right) \subseteq H \cap M$. By Proposition 4, $P_{3} \subseteq F(L)$ so $P_{3} \triangleleft O_{p^{\prime}}(L) N_{L}\left(P_{2}\right)=L$ whence $P_{3}=1$ by (*). Burnsides' Transfer Theorem yields that $L$ has a normal $p$-complement. Thus $\left[P_{2}, \tilde{Q}\right]=1$ so that $\tilde{P} \subseteq P_{2} \subset P_{1}$ (by the choice of $q$ ). If $Q_{2}$ is the $\Sigma$-invariant Sylow $q$-subgroup of $L, Q_{2} \neq Q_{1}$ (by the choice of $c$ ) and so there exists $d \in\left\langle b_{0}, a_{0}\right\rangle$ with $C_{Q_{2}}(d) \neq Q_{1}$.

Let $F$ be a maximal $\Sigma$-invariant subgroup of $G$ containing $C_{G}(d)$ (note that $H \neq F \neq M)$. As $C_{P}(d)=P_{1}$ and $P$ is non-abelian, arguing as above we conclude that $P_{1}$ is a Sylow $p$-subgroup of $F, P^{*}=N_{F}\left(P_{1}\right)^{\prime} \cap P_{1} \triangleleft F$ and $P^{*} \subseteq P_{0}$. Since $\tilde{P} \subset P_{1}, 1 \neq\left[c, P_{1}\right] \subseteq P^{*}$ which contradicts (*). 
We have proved that $H \neq P(H \cap M)$. Use the bar convention for $H / C_{H}\left(P_{1}\right)$. Since $C_{G}(\sigma) \subseteq H \cap M$, there exists $\bar{X} \cong Z_{q} \times Z_{q}$, $\Sigma$-invariant with $[\bar{X}, \sigma]=\bar{X}$. Put $\Omega=\Omega_{1}\left(Z_{2}(P)\right) \subseteq P_{1}$. Recall that $C_{\Omega}(\sigma)=\left\langle a_{0}\right\rangle$ and $1 \neq[\Omega, \sigma] \subseteq P_{0}$. If $[\bar{X}, \Omega] \neq 1, \sigma$ has a fixed point on $[\bar{X}, \Omega]$, which contradicts $C_{\Omega}(\sigma)=\left\langle a_{0}\right\rangle$. Thus $[\Omega, \bar{X}]=1$ so that $C_{P_{0}}(\bar{X}) \neq 1$. Thus the centralizer of the $\Sigma$-invariant subgroup $C_{P_{0}}(\bar{X})$ does not lie in $M$, which contradicts $(*)$. This completes the proof of the lemma.

Lemma 5. If $p \in \mathscr{P}$ then $C_{Z}(a)=1$. Further, if $z \in Z$, then $C_{M}(z)=$ $T \times O(M)=C_{G}(z)$.

Proof. Suppose $C_{Z}(a) \neq 1$ and let $U=\left\langle u, u^{\sigma}\right\rangle$ be a $\Sigma$-invariant four group in $C_{Z}(a)$ with $u \in C_{Z}(\pi)$. Suppose that $P_{0}=O(M) \cap P_{1} \neq 1$. Let $L$ be a maximal $\Sigma$-invariant subgroup of $G$ containing $N_{G}\left(P_{1}\right), P_{2}$ a Sylow $p$-subgroup of $L$ and $R=N_{P_{2}}\left(P_{1}\right)$. Using the same argument as in the previous lemma we get that $\left|R: P_{1}\right|=p^{2}$. As $L$ has a normal 2complement we may assume that $\Sigma U$ normalizes $R / P_{1}$. By Proposition 7 , $[U, R] \subseteq P_{1}$, which contradicts Lemma 2(iii). Hence $P_{0}=1$ and $P_{1}=\langle a\rangle$.

Let $A=C_{P}(a)$. As $U$ acts on $N_{P}(A) / A$ and $C_{P}(U)=\langle a\rangle, \sigma$ has a nontrivial fixed point on $N_{P}(A) / A$ if $N_{P}(A) \neq A$. However $a \in C_{G}(\sigma)$ is abelian, so we conclude that $A=P$; that is, $\langle a\rangle \subseteq Z(P)$.

Let $N=N_{G}(Z(J(P)))$. We will show that $N \subseteq C_{G}(a)$. As $N \nsubseteq M$, a maximal $\Sigma$-invariant subgroup of $G$ which contains $N$ has a normal 2complement (Lemma 2(ii)). Thus $N$ has a normal 2-complement, and so $N=O(N) \cdot(T \cap N)$. Now $[T \cap N,\langle a\rangle] T \cap Z(J(P))=1$ and $T \cap N \subseteq$ $C_{T}(a)$. A maximal $\Sigma$-invariant subgroup of $G$ containing $C_{G}(a)$ also has a normal 2-complement. Hence $C_{G}(a)=O\left(C_{G}(a)\right) \cdot C_{T}(a)$. The Frattini argument yields that $C_{T}(a) \subseteq N$. In particular $U \subseteq T \cap N=C_{T}(a)$. By Proposition 4, $O(N)^{\prime} \subseteq F(O(N)) \subseteq C_{N}(a)$, whence $C_{O(N)}(a) \triangleleft O(N)$. Also, Lemma 2(iii) yields $C_{O(N)}(U) \subseteq M \cap O(N) \subseteq C_{N}(a)$. Therefore, if $O(N) / C_{O(N)}(a) \neq 1, \sigma$ has a non-trivial fixed point on $O(N) / C_{O(N)}(a)$. This contradicts $C_{G}(\sigma) \subseteq C_{G}(a)$ and we have shown that $N \subseteq C_{G}(a)$. It follows now from Proposition 3 (ii) that $\langle a\rangle$ is weakly closed in $P$ with respect to $G$.

Since $P \neq\langle a\rangle$ and $u \sim u^{\sigma} \sim u^{\sigma^{2}}$ in $N_{G}(P) \cdot \Sigma, C_{P}(U) \neq\langle a\rangle$. Now $C_{P}(U)=P \cap M=\langle a\rangle$ so $\pi$ inverts $C_{P /\langle a\rangle}(u)$ by Proposition 7. Hence $\pi$ inverts $C_{P}(u)$ (as $a \in C_{P}(\sigma)$ ) and in particular, $C_{P}(u)$ is abelian. The fact that $\langle a\rangle$ is weakly closed in $C_{P}(u)$ means that $C_{P}(u)$ is a Sylow $p$ subgroup of $C_{G}(u)$. Further, $N_{G}(\langle a\rangle)=C_{G}(a)$ as $\sigma$ must centralize the cyclic group $N_{G}(\langle a\rangle) / C_{G}(a)$. The transfer theorem [4, Theorem 7.4.4] gives 
$a \notin O^{p}\left(C_{G}(u)\right)$. Since $T \subseteq C_{G}(u)$, the Frattini argument yields $C_{G}(u)=$ $\sigma^{p}\left(C_{G}(u)\right) \cdot\langle a\rangle$.

For any $q \in \mathscr{P}-\{p\}$, let $Q_{1}$ be the $\Sigma$-invariant Sylow $q$-subgroup of $M$, $Q_{1}=\langle d\rangle \times Q_{0}, d \in C_{M}(\sigma), Q_{0}=O(M) \cap Q_{1}$. Suppose that $\left[d, C_{T}(a)\right] \neq 1$. As $d \in C_{G}(a)$, this implies that the maximal $\Sigma$-invariant subgroup of $G$ containing $C_{G}(a)$ does not have a normal 2-complement. Thus $C_{T}(d)=$ $C_{T}(a)$ and $d \in C_{G}(u)$. The same argument as above yields that $C_{G}(u)=$ $O^{q}\left(C_{G}(u)\right)\langle d\rangle$. As $P_{0}=1, M$ contains an abelian Hall $\mathscr{P}$-subgroup $B \subseteq$ $C_{M}(\sigma)$, and $M=(T \times O(M)) \cdot B$. Also $C_{G}(u)$ has a normal subgroup $Y$ with $Y \cap M=T \times O(M)$ and $C_{G}(u)=Y \cdot B$.

Since $N_{Y}(Z(T))=N_{Y}\left(J_{e}(T)\right)=N_{Y}(T)=T \times O(M)$, Proposition 10 yields that $Y$ has a normal 2-complement. As $\langle a\rangle$ is a Sylow $p$-subgroup of $M$, the Frattini argument yields that $\langle a\rangle T \subseteq N_{Y}(\tilde{P})$ for some Sylow $p$ subgroup $\tilde{P}$ of $O(Y)$. The weak closure of $\langle a\rangle$ in $P$ forces $[\langle a\rangle, \tilde{P}]=1$. However $1 \neq[\langle a\rangle, T]$ must centralize $\tilde{P} \neq 1$, a contradiction of Lemma 2(iii). We have proved that $C_{Z}(a)=1$.

It remains to show that $C_{G}(z)=C_{M}(z)$ for $z \in Z$. As $C_{M}(z)=T \times$ $O(M)$ and $N_{G}\left(J_{e}(T)\right)=M$, Proposition 10 yields that $C_{G}(z)$ has a normal 2-complement $K$. We claim that $|Z| \geq 64$. Indeed $\langle\sigma\rangle \times\left\langle a_{0}\right\rangle$ acts fixedpoint-free on $Z$. If $|Z|=16$ then $a_{0}$ has order 5 . However $\pi$ inverts $\left\langle\sigma, a_{0}\right\rangle$ whereas $G L(4,2) \cong A_{8}$ has no dihedral group of order 30 . Thus $|Z| \geq 64$, and $\left|C_{Z}(\pi)\right| \geq 8$. If $K \neq O(M)$ there exists a four group $\langle t, u\rangle \subseteq$ $C_{Z}(\pi)$ with $C_{K}(\langle t, u\rangle) \nsubseteq O(M)$. As $\left\langle t^{\sigma}, u^{\sigma}\right\rangle$ acts on this group we may assume that $C_{K}\left(\left\langle t, t^{\sigma}\right\rangle\right) \nsubseteq O(M)$. However $\left\langle t, t^{\sigma}\right\rangle$ is $\Sigma$-invariant (recall that $\left.t \in C_{Z}(\pi)\right)$. This contradicts Lemma 2(iii). Thus $C_{G}(z)=T \times O(M)$ and the lemma is proved.

LemMa 6. The subgroup $C_{G}(\pi)$ has a normal 2-complement.

Proof. We begin with two remarks. First, if $1 \neq X \subseteq Z(T)$ then $N_{G}(X) \subseteq N_{G}(T) C_{G}(X) \subseteq M$ (by the Frattini argument and Lemma 5). Second, if $T_{\pi}=C_{T}(\pi)$, we have that $T_{\pi}$ is a Sylow 2-subgroup of $C=C_{G}(\pi)$. (If not, $T_{\pi} \subset T^{g}$ for some $\langle\pi\rangle$-invariant Sylow 2-subgroup $T^{g}$ of $G$ with $T^{g} \cap C$ a Sylow 2-subgroup of $C$. As there exists $u \in T_{\pi} \cap Z, O(M)=$ $O\left(M^{g}\right)=1$. Further, all involutions in $\pi T$ are conjugate in $T$ and so, $\pi, \pi^{g}$ are conjugate in $N_{G}\left(T^{g}\right)=M^{g}$. That is, there exists $h \in M^{g}$ with $\pi=\pi^{g h}$. Therefore $T_{\pi}^{g h}=C \cap T^{g h}=C \cap T^{g}$ and so $T_{\pi}$ is (conjugate to) a Sylow 2-subgroup of $C$.)

Now suppose that $C$ does not have a normal 2-complement. We note that $N_{G}\left(Z\left(T_{\pi}\right)\right)=N_{G}\left(T_{\pi}\right) \cdot C_{G}\left(Z\left(T_{\pi}\right)\right) \subseteq N_{G}\left(T_{\pi}\right) \cdot T$ as $C_{G}\left(Z\left(T_{\pi}\right)\right) \subseteq T \times O(M)$ by Lemma 5. Therefore we have $N_{C}\left(T_{\pi}\right)=N_{C}\left(Z\left(T_{\pi}\right)\right)$. It follows from 
Proposition 10 that either $N_{C}\left(T_{\pi}\right) \neq C_{C}\left(T_{\pi}\right) \cdot T_{\pi}$ or there exists $S_{\pi} \triangleleft T_{\pi}$, $T_{\pi} / S_{\pi}$ cyclic and $N_{C}\left(S_{\pi}\right) / S_{\pi} C_{C}\left(S_{\pi}\right)$ has a non-trivial normal 2-complement. Let $S$ denote either $T_{\pi}$ or $S_{\pi}$ and let $x$ be an element of odd order in $N_{C}(S)-M$. (We can choose $x \notin M$ as $C_{M}(\pi)=T_{\pi} \times C_{O(M)}(\pi)$.)

As $x$ normalizes $S^{\prime} \subseteq Z(T)$, the first remark (at the beginning of the proof) yields that $S$ is abelian. Since $Z \cap S \neq 1, C_{G}(S)=C_{T}(S) \times O(M)$. Let $A=C_{T}(S)$. As $x$ normalizes $O\left(C_{G}(S)\right)=O(M), O(M)=1$ and $C_{G}(S)=A \supseteq S$.

We have $x \in N_{G}(A)$ and therefore, arguing as for $S$, we get that

$$
\left(O_{2}\left(N_{G}(A)\right)\right)^{\prime}=1 .
$$

Thus $A=O_{2}\left(N_{G}(A)\right)=C_{G}(A)$. Set $N=N_{G}(A)$ and apply the bar convention to $\bar{N}=N / A$. If $N(\bar{T}) \neq C(\bar{T})$ there exists $d$ of order $q$, for some $q \in \mathscr{P}$ with $d \in C_{M}(\sigma)$ and $[\bar{d}, \bar{T}] \neq 1$. Since $[d, T] \cap C_{T}(d)=1$ (recall that $\left.C_{Z}(d)=1\right),\langle d, \pi\rangle$ acting on $[d, T]$ satisfies the assumptions of Proposition 9. Therefore $C_{[d, T]}(\pi)$ covers $C_{[d, T] /[d, T] \cap A}(\pi)$. As

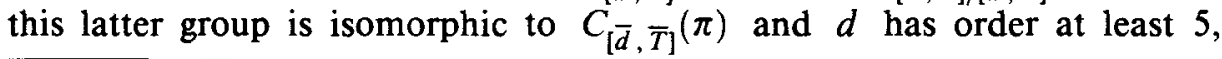
$\overline{C_{[d, T]}(\pi)} \subseteq \bar{T}_{\pi}$ is non-cyclic. This contradicts the fact that $T_{\pi} / S$ is cyclic (recall that $S \subseteq A)$. We conclude that $N(\bar{T})=C(\bar{T})$ and so $\bar{N}$ has a normal 2-complement $\bar{K} \neq 1$.

If $\bar{T}$ is not cyclic, let $\left\langle\bar{t}_{1}, \bar{t}_{2}\right\rangle$ be a four group in $\bar{T}$. We may assume that $\left[\bar{t}_{1}, C_{\bar{K}}\left(\bar{t}_{2}\right)\right]=\bar{K}_{0} \neq 1$. Now $\left[\bar{t}_{2}, A\right]=A_{0} \subseteq Z(T)$ so $\left[\bar{t}_{1}, A_{0}\right]=1$. Hence $\left[\bar{K}_{0}, A_{0}\right]=1$, which contradicts Lemma 5 . If $\bar{T}$ is cyclic, let $\langle\bar{t}\rangle=\Omega_{1}(\bar{T})$. Clearly $\left|\left[\bar{t}, \Omega_{1}(A)\right]\right| \geq 4$ as $\bar{t}$ inverts an element of odd order in $\bar{K}$. This forces $J_{e}(T) \subseteq A$ and $N \subseteq M$, a contradiction. The lemma is proved.

We are now in a position to complete the proof of the theorem. By Lemma 2(i), $\mathscr{P} \neq \varnothing$, so let $p \in \mathscr{P}$. By Proposition $6, P_{\pi}=C_{P}(\pi) \neq 1$. As $a$ is fixed-point-free on $Z$ (recall that $P_{1}=\langle a\rangle \times P_{0}, P_{1}$ a Sylow $p$-subgroup of $M)$, there exists a four group $\left\langle u_{1}, u_{2}\right\rangle \subseteq C_{Z}(\pi)$. Lemma 6 shows that $\left\langle u_{1}, u_{2}\right\rangle$ normalizes a Sylow $p$-subgroup $\tilde{P}$ of $C=C_{G}(\pi)$. Since $C_{G}(z)=$ $T \times O(M)$ for $z \in Z^{\#}$, it follows that $\tilde{P} \subseteq O(M)$. The same argument as in the (second) remark at the beginning of the proof of Lemma 6 yields that $C \cap P_{1}=C \cap P_{0}$ is a Sylow $p$-subgroup of $C_{M}(\pi)$. As $\tilde{P} \subseteq O(M)$, $C \cap P_{0}$ is a Sylow $p$-subgroup of $C$. Thus $[\sigma, P]=\left[\sigma, P_{0}\right]$ (if $[\sigma, P] \nsubseteq P_{1}$ then $P_{\pi} \nsubseteq P_{1}$ ). If $H$ is a maximal $\Sigma$-invariant subgroup of $G$ containing $N_{G}([\sigma, P])$, then $H \supseteq\langle T, P\rangle$ so $H \neq M$ (Lemma 3(ii)). As $p \in \mathscr{P}$ we have $1 \neq\left[P_{1}, T\right] \subseteq T$. Thus $H$ does not have a normal 2-complement, against Lemma 2(ii). This contradiction completes the proof of the theorem. 


\section{References}

[1] B. Dolman, Ph. D. Thesis, University of Adelaide, 1984.

[2] G. Glauberman, 'A characteristic subgroup of a p-stable group', Canad. J. Math. 20 (1968), 1101-1135.

[3] G. Glauberman, Factorizations in local subgroups of finite groups, (CBMS 33, Amer. Math. Soc., Providence, R.I., 1977).

[4] D. Gorenstein, Finite groups, (Harper and Row, New York, 1968).

[5] B. Huppert and N. Blackburn, Finite groups II, (Springer-Verlag, Berlin, Heidelberg, New York, 1982).

[6] R. P. Martineau, 'Elementary abelian fixed-point-free automorphism groups', Quart. $J$. Math. Oxford Ser. (2) 23 (1972), 205-212.

[7] B. Rickman, 'Groups which admit a fixed-point-free automorphism of order $p^{2}, J$. Algebra 59 (1979), 77-171.

[8] P. J. Rowley, 'Solubility of finite groups admitting a fixed-point-free abelian automorphism group of square-free exponent rs', Proc. London Math. Soc. (3) 37 (1978), 385421.

[9] E. Shult, 'On groups admitting fixed-point-free abelian operator groups', Illinois J. Math. 9 (1965), 701-720.

[10] E. Shult, "Nilpotence of the commutator subgroup in groups admitting fixed point free operator groups', Pacific J. Math. 17 (1966), 323-347.

University of Adelaide

Adelaide, S.A. 5001

Australia 